\title{
Histological changes in the human esophagus following triamcinolone injection to prevent esophageal stricture after endoscopic submucosal dissection
}

\author{
Yudai Kawamura ${ }^{1,2} \cdot$ Kenro Kawada $^{1} \cdot$ Takashi $_{\text {Ito }}{ }^{3} \cdot$ Katsumasa Saito $^{1} \cdot$ Naoto Fujiwara $^{1} \cdot$ Takuya Okada $^{1}$. \\ Akihiro Hoshino $^{1} \cdot$ Yutaka Tokairin $^{1,2}$ - Yasuaki Nakajima ${ }^{4}$ Tatsuyuki Kawano ${ }^{5}$. Masanori Tokunaga ${ }^{1}$. \\ Yusuke Kinugasa ${ }^{1}$
}

Received: 20 August 2020 / Accepted: 1 February 2021 / Published online: 2 March 2021

(c) The Author(s) 2021

\begin{abstract}
Background Locoregional steroid injection prevents post-endoscopic submucosal dissection (ESD) esophageal stricture, but histological changes that occur following steroid injection in the human esophagus are unclear. This study investigated the histopathological characteristics caused by locoregional triamcinolone acetonide (TA) injection using human esophagectomy specimens.

Methods From January 2014 to December 2019, among 297 patients (373 lesions) who underwent esophageal ESD, 13 patients who underwent additional esophagectomy after ESD were examined. Seven patients (TA group) with wide excisions were injected with TA after ESD and another six patients (Non-TA group) with smaller tumors were not injected with TA. The clinical background of these patients and histopathological features of ESD ulcer scar obtained from esophagectomy specimens were retrospectively investigated.

Results The circumferential rate of ESD excision was more than three-quarters in all cases in the TA group, whereas it was less than three-quarters in the Non-TA group. No other statistical difference in the clinical background was found between the two groups. The subepithelial fibrous tissue of the ESD ulcer scar in the TA group was significantly thinner than that in the Non-TA group $(P<0.05)$. There was no significant difference in the thickness of the regenerated epithelium and muscularis propria layer of the ESD ulcer scar.

Conclusions Histological finding of thinning of the subepithelial fibrous tissue of ESD ulcer scar in the human esophagus after TA injection was obtained. This suggests that TA suppresses the proliferation of the fibrous tissue of the subepithelial layer to help prevent esophageal stricture after widespread ESD in the human esophagus.
\end{abstract}

Keywords Triamcinolone acetonide $\cdot$ Esophageal cancer $\cdot$ Esophageal stenosis $\cdot$ Esophagectomy

Kenro Kawada

kawada.srg1@tmd.ac.jp

1 Department of Gastrointestinal Surgery, Graduate School of Medical and Dental Sciences (Medicine), Tokyo Medical and Dental University, 1-5-45 Yushima, Bunkyo-ku, Tokyo 113-8510, Japan

2 Department of Surgery, Tokyo Metropolitan Health and Hospitals Corporation Toshima Hospital, Tokyo, Japan

3 Department of Human Pathology, Graduate School of Medical and Dental Sciences (Medicine), Tokyo Medical and Dental University, Tokyo, Japan

4 Department of Surgery, Edogawa Hospital, Tokyo, Japan

5 Department of Surgery, Soka Municipal Hospital, Saitama, Japan

\section{Introduction}

Esophageal cancer is the ninth most common malignancy in the world [1]. For the treatment of superficial esophageal cancer, with no risk of lymph node metastasis, endoscopic submucosal dissection (ESD) was developed in Japan and has spread worldwide with the progress of endoscopic diagnostic and treatment techniques [2,3]. Compared to endoscopic mucosal resection (EMR), ESD makes it possible to resect specimen en bloc regardless of tumor size, leading to a more precise pathological diagnosis. The clinical outcomes of esophageal ESD are comparable to surgical treatment. Moreover, its risk of complications is lower than that in highly invasive esophagectomy [4-7]. However, esophageal 
ESD with a wide excision range is associated with a high occurrence of esophageal strictures [8]. Esophageal stricture after widespread ESD is a serious complication that lowers the patient's quality of life and requires frequent balloon dilatation [9].

Previous studies showed that local steroid injection into the ESD ulcer prevented post-ESD stricture [10-12]. In our institution, when the circumferential rate of ESD ulcer is more than three-quarters, triamcinolone acetonide (TA) is injected locally into the artificial ulcer layer and good clinical results are obtained.

Although TA local injection after widespread ESD may suppress fibrosis and is clinically effective in preventing esophageal stricture, it is not well understood how TA local injection causes histological changes in the human esophagus. The aim of this study was to retrospectively investigate the histological features, such as suppression of fibrosis caused by local TA injection, using the human esophagectomy specimens obtained from the patients who underwent additional surgery after ESD.

\section{Patients and methods}

\section{Patients}

In total, 297 patients with 373 superficial esophageal cancers were treated with ESD at Tokyo Medical and Dental University Hospital from January 1, 2014 to December 31, 2019. Thirteen of these patients (2.95\%) with 14 lesions underwent additional radical esophagectomy based on the subsequent pathological diagnosis of the specimens obtained by ESD. Of these, seven patients with seven lesions had received local injection of TA (Kenacort-A $40 \mathrm{mg} / 1 \mathrm{~mL}$; Bristol-Myers Squibb Co., Tokyo, Japan) to the superficial layer of the residual submucosal tissue of the ESD ulcer bed to prevent esophageal stricture due to extensive resection (circumferential rate $\geq 3 / 4$ ) during ESD. The remaining six patients with seven lesions were not injected with TA after ESD because the ulcer was small (circumferential rate $<3 / 4$ ). The esophagectomy specimens obtained from these 13 patients were histologically examined retrospectively. All of these patients had not received any treatment, such as chemotherapy or radiation therapy, prior to ESD.

\section{TA injection}

Except for one case where TA was injected the day after ESD, a single session of locoregional TA injection was performed immediately after ESD in all cases. The TA injection procedure is shown in Fig. 1. TA (Kenacort-A $40 \mathrm{mg} / 1 \mathrm{~mL}$ ) was injected into the superficial layer of the ESD ulcer bed, using a 25-gauge or 26-gauge endoscopic needle at $0.1 \mathrm{~mL}$ each time for a total of $1 \mathrm{~mL}$. Since there have been reports of periesophageal abscess formation or delayed esophageal perforation after TA injection [13-15], we were careful not to inject deeper than the muscularis propria layer.

\section{Clinical and histopathological survey}

The clinical background of these 13 patients was retrieved from their medical records and endoscopic reports. We investigated sex, age, tumor location, interval between ESD and operation, stricture rate, macroscopic type of tumor, tumor histology, depth of tumor invasion, lymphovascular invasion, dissection margin, size of ESD specimen, tumor remnant, lymph node metastasis, and size of ESD scar. In addition, the histopathological evaluation was performed retrospectively using the radical esophagectomy specimens, in which the ESD ulcer scars existed, to determine characteristic findings such as suppression of fibrosis in ulcer scars attributable to TA injection. The esophagectomy specimens were cut into 3- to 4-mm slices, fixed with formalin, embedded in paraffin, and then sliced into 3 - $\mu$ m-thick sections for
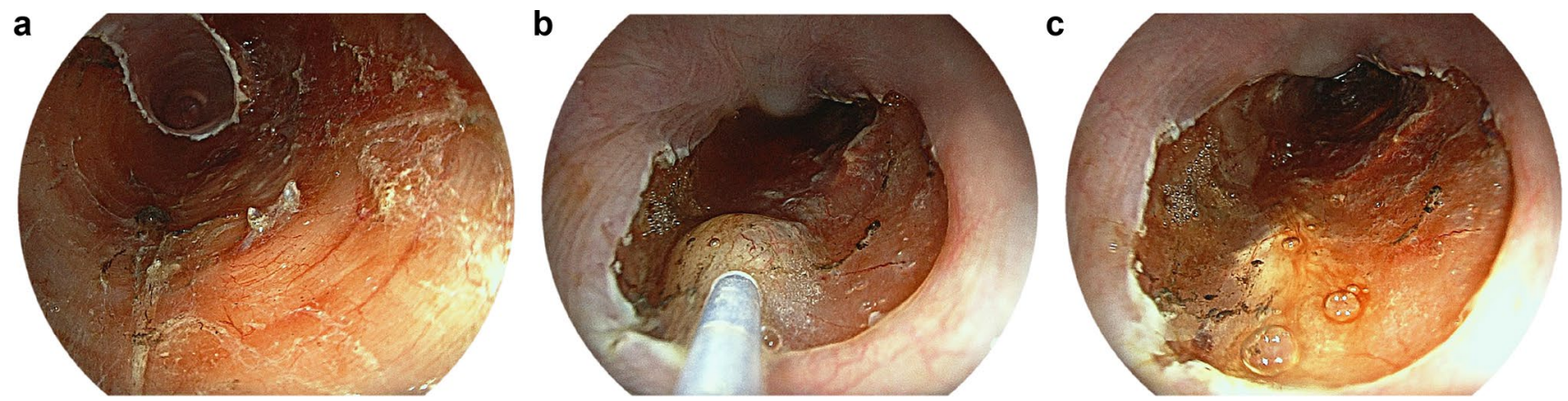

Fig. 1 Representative endoscopic appearance of TA injection after ESD. a A subcircumferential artificial ulcer was created after ESD. The muscularis propria layer was found under the residual submucosal layer. b After the ESD, TA was injected into the submucosal layer of the ulcer site, being careful not to inject deeper than the muscularis propria layer. $\mathbf{c}$ White suspended liquid was stored in the residual submucosal tissue. ESD endoscopic submucosal dissection, $T A$ triamcinolone acetonide 
hematoxylin-eosin (HE) and Elastica van Gieson (EVG) staining. These histopathological specimens had been prepared at the Department of Pathology, Tokyo Medical and Dental University Hospital, and were used for pathological diagnosis. Masson trichrome (MT) staining and desmin immunohistochemical staining were performed for more detailed histological observation. The primary antibody to desmin (clone D33) was obtained from MONOSAN (Concord, CA, USA). The antibody was used at a dilution of 1:50. Immunohistochemical analysis was performed using a Leica Bond III automated immunostainer (Leica, Bannockburn, IL, USA). BOND Epitope-Retrieval Solution 2 (AR9640) was used for 20 min before application of the primary antibody (15 min). Detection was done using Leica Bond-detection kit (DS9800).

Histopathologically, ESD ulcer scars were defined as the area of the muscularis mucosal defects in esophagectomy specimens. Regenerated epithelium (ReEp) and fibrous tissue proliferation were observed there. The layer between the ReEp and the muscularis propria (MP) in the ulcer scar is referred to as the subepithelial layer or subepithelial fibrous tissue (SF).

All sections of the ulcer scar were observed under a microscope to extract histological characteristics, focusing on the degree of fibrosis. The thickness of each layer (ReEp, SF and MP) in the esophageal wall at the center of the ulcer scar were measured using the software CellSens (Olympus, Tokyo, Japan). These clinicopathological characteristics of patients who received TA injection after ESD (TA group) were compared with those who did not receive TA injection (Non-TA group).

\section{Statistical analyses}

All statistical analyses were performed with EZR, version 1.42 (Saitama Medical Center, Jichi Medical University, Saitama, Japan), which is a graphical user interface for $\mathrm{R}$ (The R Foundation for Statistical Computing, Vienna, Austria). More precisely, it is a modified version of $\mathrm{R}$ commander designed to add statistical functions frequently used in biostatistics [16]. Categorical data were analyzed with Fischer's exact test, while continuous data were analyzed with Mann-Whitney $U$ test. The differences with $P<0.05$ (two-sided) were considered statistically significant.

\section{Results}

\section{Baseline characteristics of the patients and tumors}

Table 1 shows the baseline characteristics of the patients and tumors analyzed. The study included 14 lesions of 13 patients (12 men and 1 woman) who underwent additional esophagectomy after ESD without prior treatment. The patients were aged from 54 to 78 years (median 65 years). Twelve cases had squamous cell carcinoma (SCC), and there was one case each of basaloid squamous cell carcinoma (BSCC) and carcinosarcoma. Additional esophagectomies in the TA group were performed because of pT1a-MM with ly1 (one case), pT1a-MM with VM1 (one case), pT1b-SM1 with a positive stump (one case), and pT1b-SM2 (four cases). In the Non-TA group, one case had pT1a-MM with ly1/v1, one case had multiple lesions (pT1a-LPM and pT1b-SM2), and the remaining cases had pT1b-SM2, so they underwent additional esophagectomy. No statistically significant differences were found in sex, age, tumor location, the interval between ESD and operation, stricture rate, macroscopic type of tumor, tumor histology, depth of tumor invasion, lymphovascular invasion, dissection margin, tumor remnant, and lymph node metastasis between the two groups. In all cases in the TA group, the circumferential rate of ESD excision was more than three-quarters, whereas it was less than three-quarters in all cases in the Non-TA group. The maximum length of the ESD specimen was longer in the TA group (median $60 \mathrm{~mm}$, range 35-75 mm) than in the Non-TA group (median $34 \mathrm{~mm}$, range $17-48 \mathrm{~mm}, P=0.007$ ). The size of the post-ESD ulcers was significantly larger in the TA group (median $55 \mathrm{~mm}$, range $23-72 \mathrm{~mm}$ ) than in the NonTA group (median $25 \mathrm{~mm}$, range $10-40 \mathrm{~mm}, P=0.015$ ). Detailed clinicopathological findings of all cases are given in supplementary Table 1.

\section{Clinical course}

One case in the TA group required balloon dilatation due to post-ESD esophageal strictures before additional esophagectomy. On the contrary, no post-ESD stricture occurred in the Non-TA group. No other complications due to ESD or local TA injection such as intraoperative or delayed perforation were reported.

\section{Histopathological findings}

Histopathological findings of the esophagectomy specimens were obtained through retrospective microscopic observation primarily using HE-stained and EVG-stained specimens and supplemented with MT-stained and desmin-stained histopathological examination. The border of the ESD ulcer scar was defined as the disrupted part of the MM. Representative findings of the TA group cases are shown in Fig. 2.

The ulcer scar was covered with the ReEp. In the TA group, overall, the SF of the ulcer scar area was thinned. The proliferation of fibrous tissue in the subepithelial layer was observed in all cases. Of the seven TA group cases, five cases demonstrated a very thin subepithelial layer over a wide area. There was an area where the ReEp was in contact 
Table 1 Baseline clinicopathological characteristics of the patients and tumors analyzed

\begin{tabular}{|c|c|c|c|}
\hline & $\begin{array}{l}\text { TA group } \\
7 \text { cases, } 7 \text { lesions }\end{array}$ & $\begin{array}{l}\text { Non-TA group } \\
6 \text { cases, } 7 \text { lesions }\end{array}$ & $P$ value \\
\hline Sex, $n(\%)$ & & & 0.462 \\
\hline Male & $7(100)$ & $5(83.3)$ & \\
\hline Female & $0(0)$ & $1(16.7)$ & \\
\hline Age, years, median (range) & $65(64-78)$ & $63(54-72)$ & 0.059 \\
\hline Tumor location, $n(\%)$ & & & 0.592 \\
\hline $\mathrm{Ce}$ & $1(14.3)$ & $0(0)$ & \\
\hline UtMt & $1(14.3)$ & $0(0)$ & \\
\hline Mt & $2(28.6)$ & $5(71.4)$ & \\
\hline MtLt & $1(14.3)$ & $0(0)$ & \\
\hline LtMtAe & $1(14.3)$ & $0(0)$ & \\
\hline $\mathrm{Lt}$ & $1(14.3)$ & $1(14.3)$ & \\
\hline $\mathrm{Ae}$ & $0(0)$ & $1(14.3)$ & \\
\hline $\begin{array}{l}\text { Number of days from ESD to operation, } \\
\text { Median (range) }\end{array}$ & $85(63-131)$ & $98(71-190)$ & 0.391 \\
\hline Stricture after ESD, $n(\%)$ & $1(14.3)$ & $0(0)$ & 1 \\
\hline \multicolumn{4}{|l|}{ ESD specimen } \\
\hline Macroscopic type of tumor, $n(\%)$ & & & 1 \\
\hline Elevated & $4(57.1)$ & $3(42.9)$ & \\
\hline Flat/depressed & $3(42.9)$ & $4(57.1)$ & \\
\hline Histology, $n(\%)$ & & & 0.462 \\
\hline $\mathrm{SCC}$ & $5(71.4)$ & $7(100)$ & \\
\hline Others & $2(28.6)$ & $0(0)$ & \\
\hline Depth of tumor invasion, $n(\%)$ & & & 1 \\
\hline pT1a & $2(28.6)$ & $2(28.6)$ & \\
\hline pT1b & $5(71.4)$ & $5(71.4)$ & \\
\hline Lymphovascular invasion positive, $n(\%)$ & $5(71.4)$ & $6(85.7)$ & 1 \\
\hline Dissection margin positive, $n(\%)$ & $5(71.4)$ & $2(28.6)$ & 0.286 \\
\hline Size of specimen, mm, median (range) & $60(35-75)$ & $34(17-48)$ & 0.007 \\
\hline \multicolumn{4}{|l|}{ Surgical specimen } \\
\hline Remnant of tumor, $n(\%)$ & $2(28.6)$ & $1(14.3)$ & 1 \\
\hline Lymph node metastasis positive, $n(\%)$ & $2(28.6)$ & $2(33.3)$ & 1 \\
\hline Size of scar, mm, median (range) & $55(23-72)$ & $25(10-40)$ & 0.015 \\
\hline
\end{tabular}

Italic values indicate significance of $p$ value $(P<0.05)$

$\mathrm{Ce}$ cervical esophagus, $U t$ upper thoracic esophagus, $M t$ middle thoracic esophagus, $L t$ lower thoracic esophagus, Ae abdominal esophagus, $S C C$ squamous cell carcinoma, ESD endoscopic submucosal dissection, $T A$ triamcinolone acetonide with the MP layer. This characteristic finding was found only in cases in the TA group and not in the Non-TA group (Table 2).

The MP layers were generally maintained, and the circular and longitudinal muscles were clearly observed. Except for one case, changes in the edematous of the MP layer were observed. No other notable findings in the MP layer such as inflammatory cell infiltration and fibrosis were observed.

Figure 3 shows the representative histological findings of the Non-TA group. In the ulcer scar, where the MM was disrupted, thick fibrous tissue was observed throughout the layer beneath the ReEp. In all six specimens with seven ESD ulcer scars, The SF layer was relatively thick; therefore, the ReEp and the MP layer were not close to each other. In the MP layer, characteristic findings were not obtained in terms of its thickness, degree of inflammatory cell infiltration, and fibrosis.

Figure 4 shows the histology of the ESD scar in esophagectomy specimens of the representative cases in both TA and Non-TA groups. The histology is depicted by HE, MT, and desmin staining.

The disappearance of the MM and proliferation of fibrous tissue was observed between the ReEp and MP layers. In the SF, the cellularity of fibroblasts/myofibroblasts was low (a, d) and dense collagen bundles were observed (b, e). Immunostaining for desmin revealed the mixture 

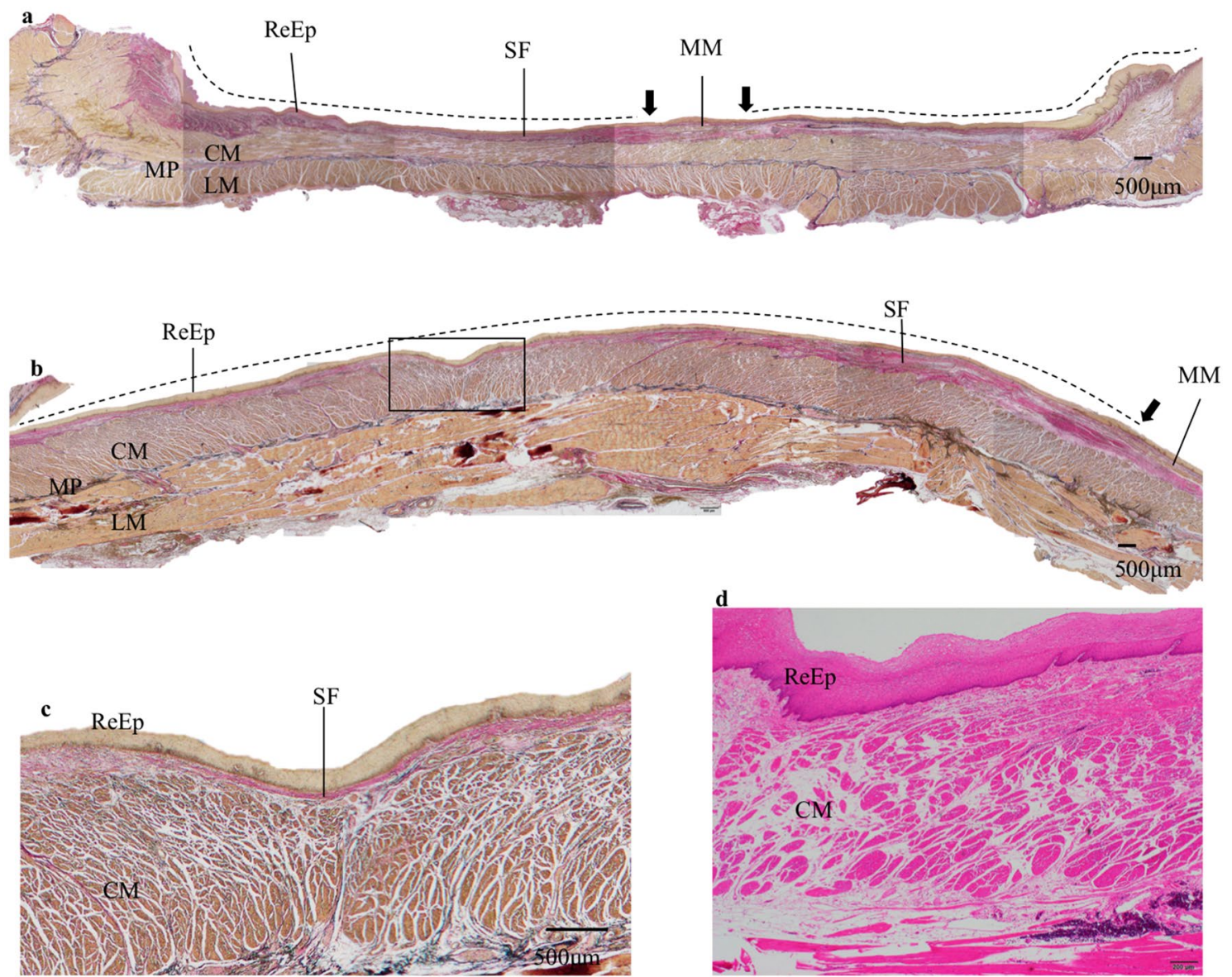

Fig. 2 Representative histological findings of the artificial ulcer in esophagectomy specimen after ESD in the TA group. a Histological findings of the esophagectomy specimen from one representative case. The black arrow indicates the border of the ESD scar because of the muscularis mucosae disruption. The dotted line indicates the ESD ulcer scar. The subepithelial fibrous tissue is relatively thin over the whole area and the regenerated epithelium and the muscularis propria are close to each other. EVG, original magnification $\times 20$. b Histological findings of the central part of an artificial ulcer in an esophagectomy specimen from another case. The black arrow indicates the disruption of muscularis mucosae, the dotted line indicates the ESD ulcer scar. The regenerated epithelium and the mus-

Table 2 Thickness of subepithelial fibrous tissue

$\begin{array}{llr}\text { TA group } & \text { Non-TA group } & P \text { value } \\ 7 \text { cases, 7 lesions } & 6 \text { cases, 7 lesions } & \end{array}$

Lack of or thinning of subepithelial fibrous tissue, $n(\%) \quad 0.021$

\begin{tabular}{lll} 
Positive & $5(71.4)$ & $0(0)$ \\
Negative & $2(28.6)$ & $7(100)$ \\
\hline
\end{tabular}

Italic value indicate significance of $p$ value $(P<0.05)$

of various amounts of smooth muscle fibers between the fibrous tissue (c, f). Thickness of the SF of the TA group was less than that of the Non-TA group. cularis propria layer are very close, and subepithelial fibrous tissue between them is barely observed. EVG, original magnification $\times 20$. c Enlarged view of the area surrounded by black squares in Fig. 1b. The subepithelial fibrous tissue is very thin and the regenerated epithelium and the muscularis propria layer are almost in contact with each other. EVG, original magnification $\times 40$. d Histological findings of another case. The regenerated epithelium and the muscularis propria layer are almost in contact with each other. HE, original magnification $\times 40$. ReEp regenerated epithelium, $M M$ muscularis mucosae, $M P$ muscularis propria, $C M$ circular muscle, $L M$ longitudinal muscle, $S F$ subepithelial fibrous tissue, $E V G$ Elastica van Gieson staining, $H E$ hematoxylin and eosin staining, $T A$ triamcinolone acetonide

Histological evaluation of the subepithelial layer is shown in Table 3. The density of collagen fibers and smooth muscle fibers was evaluated in three stages. The number of spindle-shaped cells per high power field (HPF) was also evaluated. Although there were variations among the cases, no statistically significant difference was found between the two groups. The thickness of each layer (ReEp, SF, and MP layer) in the esophageal wall at center of the ulcer scar was measured. The median thickness of the SF layer was $185 \mu \mathrm{m}$ (range $15-1151 \mu \mathrm{m}$ ) in the TA group, while it was $860 \mu \mathrm{m}$ (range $753-887 \mu \mathrm{m}$ ) in the Non-TA group. SF layer in the TA group was significantly thinner $(P=0.030)$ than the Non-TA group. No significant difference was observed 

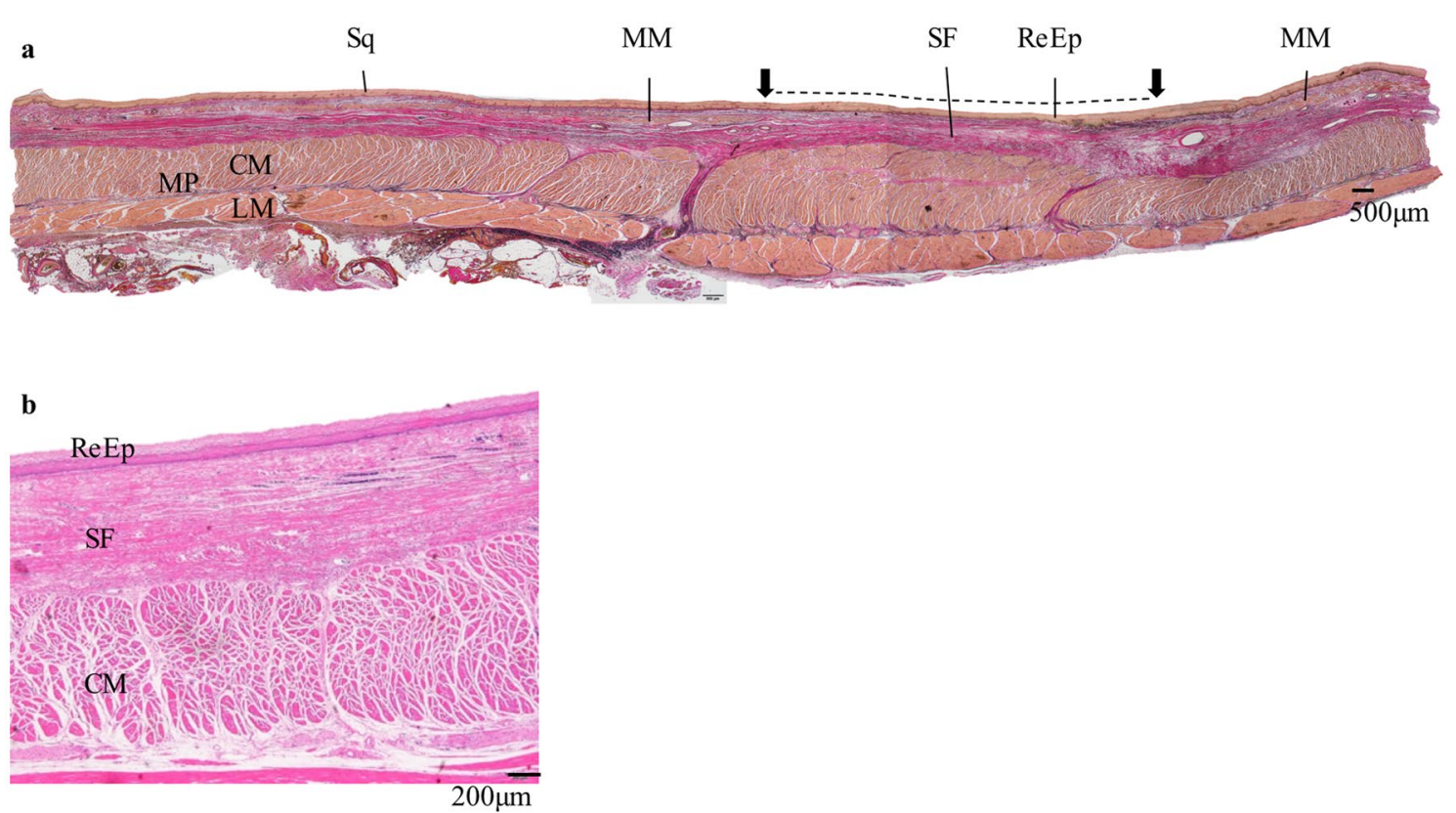

Fig. 3 Histological findings of the artificial ulcer after ESD in esophagectomy specimen of the Non-TA group. a Histological findings of the resected specimen around the artificial ulcer of one representative case. The disrupted part of the muscularis mucosae indicates the boundary of the artificial ulcer (black arrow). The dotted line indicates the ESD ulcer scar. In the artificial ulcers' scar, sufficient volume of fibrous tissue is found beneath the regenerated epithelium. The thickness of the muscularis propria layer is not very different from that of the normal esophageal wall. All other Non-TA group specimens had similar subepithelial fibrous tissue thickness.

in the thickness of the ReEp and MP layers between the two groups.

\section{Discussion}

In the present study, we investigated the histopathological characteristics caused by locoregional TA injection by using the esophagectomy specimens of patients who had undergone additional surgery after ESD. The SF of the ulcer scar in cases with TA injection was thinner than that in cases without TA injection. In five of seven TA cases, the SF of the ESD ulcer scar was almost deficient, and the MP layer and the ReEp were in contact. These findings were not observed in any of the cases without TA injection. No characteristic findings were obtained in terms of the thickness of the ReEp, degree of inflammatory cell infiltration, and thickness of the MP layer. No significant correlation was found between the size of the ESD specimen and that of the ESD ulcer scar in the esophagectomy specimens, regardless of TA injection. These histological findings suggest that locoregional TA injection suppresses the proliferation of the SF and may
EVG, original magnification $\times 20$. b Histological findings of the artificial ulcer of another case in higher magnification. Muscularis mucosae is not found in the area. Under the regenerated epithelium, thick subepithelial fibrous tissue is observed. The muscularis propria layer is thick. HE, original magnification $\times 40$. $S q$ squamous epithelium, ReEp regenerated epithelium, $M M$ muscularis mucosae, $M P$ muscularis propria, $C M$ circular muscle, $L M$ longitudinal muscle, $S F$ subepithelial fibrous tissue, $E V G$ Elastica van Gieson staining, $H E$ hematoxylin and eosin staining

contribute to the prevention of esophageal strictures after widespread ESD.

Previous studies have reported methods, such as preventive balloon dilatation, steroid administration, esophageal stent treatment, and tissue engineering, to prevent post-ESD esophageal stricture [11, 17-22]. Among these methods, steroid administration to prevent stricture included oral steroid administration, TA injection, and TA-filling method. Since Yamaguchi et al.'s first report [23], oral steroid therapy may have been considered the simplest method, but complications due to long-term use of steroids cannot be ignored. A recent study reported the usefulness of the TA-filling method [24]. In particular, locoregional TA injection therapy is widely accepted for its effectiveness and usefulness as a post-ESD stricture prevention method [10, 22, 25].

Despite TA injection, some patients still develop esophageal strictures after ESD [26, 27]. Okamoto et al. [26] reviewed 53 ESD cases (57 lesions) who received local TA injection to prevent esophageal stricture due to widespread exfoliation of more than two-thirds of the circumference. They reported that the rate of esophageal strictures was highest if the mucosal defect was more than seven-eighths of the circumference; thus, in such cases, alternative strategies, 

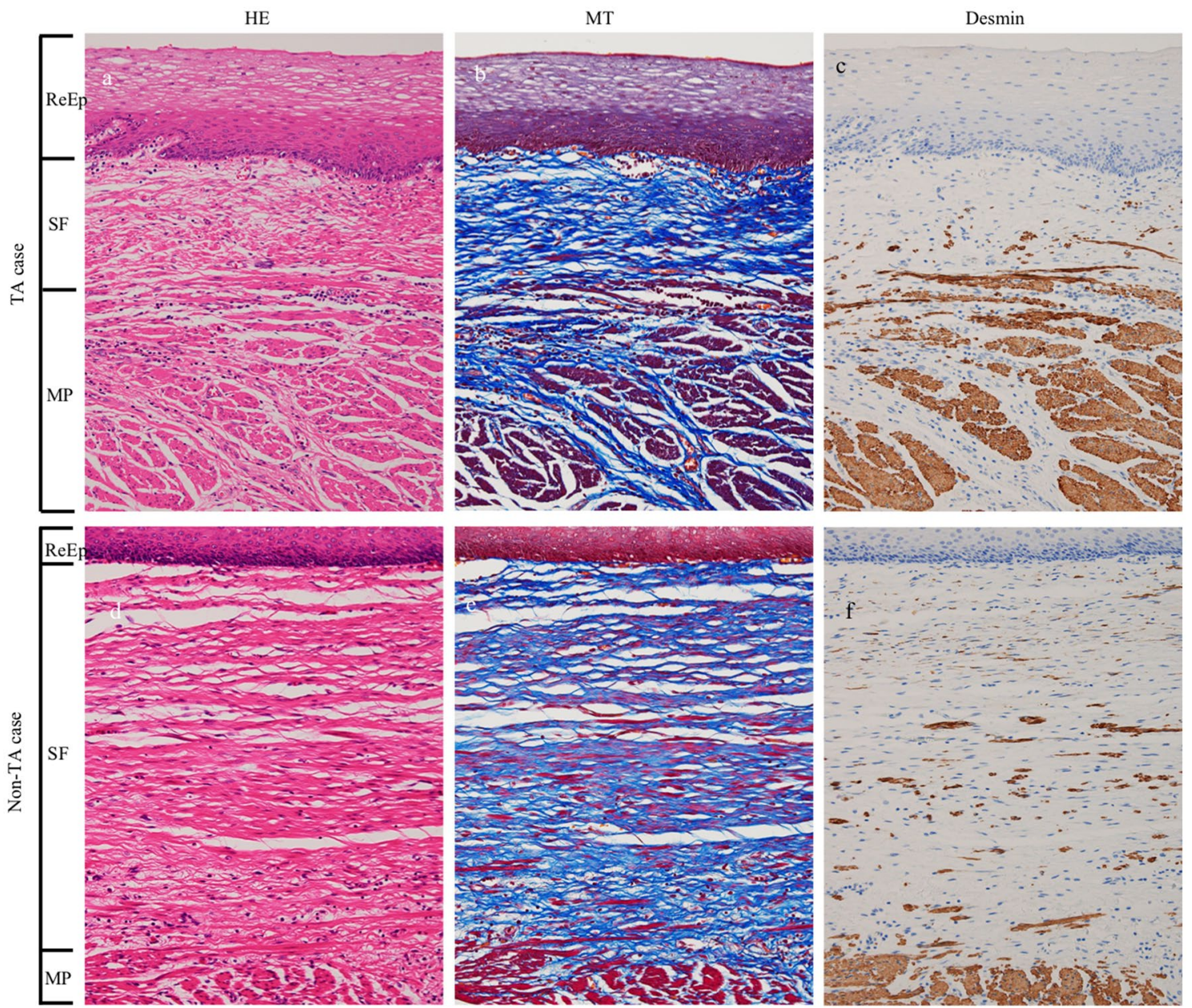

Fig. 4 Comparison of histology of the ESD scar in esophagectomy specimens of the representative cases in both TA and Non-TA group. The disappearance of muscularis mucosae and proliferation of fibrous tissue was observed between regenerated epithelium and muscularis propria. In the subepithelial fibrous tissue, the cellularity of fibroblasts/myofibroblasts is low (a, d) and dense collagen bundles were observed (b, e). Immunostaining for desmin revealed the mixture

such as application of polyglycolic acid sheets [20], are required. In our study, one patient with TA injection had post-ESD stricture and required balloon dilatation. In this case, the proliferation of the SF was strongly observed (data not shown). The observation that the exfoliation area was particularly large in this case is consistent with the findings of Okamoto et al. [26].

In the fields of dermatology and plastic surgery, it has been established that Kenacort injection is effective in treating keloids [28, 29]. Several basic histopathological studies have revealed that steroid suppresses fibroblast proliferation by controlling chemokines and cytokines [30, 31]. In contrast, to the best of our knowledge, no histological study focused on stricture-preventing effect of steroid injection in human esophagus has been conducted. of various amounts of smooth muscle fibers between the fibrous tissue $(\mathbf{c}, \mathbf{f})$. The thickness of the subepithelial fibrous tissue in the TA group was less than that in the Non-TA group. ReEp regenerated epithelium, $S F$ subepithelial fibrous tissue, $M P$ muscularis propria, $H E$ hematoxylin and eosin staining, MT Masson trichrome staining, TA triamcinolone acetonide

Honda et al. [32] performed esophageal EMR of six canine models, observed the ulcer scar histologically immediately after EMR and on postoperative days (POD) 2, 4, 7, 14, and 28, and examined the time course of histological changes during the healing process. They reported that after the inflammatory reaction in the submucosal layer caused by food, digestive juices, saliva, and reflux of gastric acid in addition to EMR itself, epithelial cells proliferated and migrated from the border on POD 7. At 1 month after EMR, the ulcer scar was covered with regenerated squamous epithelium, and myofiber atrophy and fibrosis in the muscularis propria remained.

Nonaka et al. [33] injected TA after esophageal ESD in a pig model and observed esophagectomy specimen immunohistologically over time. They reported that during the 
Table 3 Histological evaluation

\begin{tabular}{|c|c|c|c|c|c|c|}
\hline \multirow[t]{2}{*}{ No. } & \multicolumn{3}{|c|}{ Histological findings of subepithelial fibrous tissue } & \multicolumn{3}{|c|}{ Thickness of each layer at the center of the ulcer, $\mu \mathrm{m}$} \\
\hline & $\begin{array}{l}\text { Density of } \\
\text { collagen } \\
\text { fiber }^{\mathrm{a}}\end{array}$ & $\begin{array}{l}\text { Density of } \\
\text { smooth muscle } \\
\text { fiber }^{b}\end{array}$ & $\begin{array}{l}\text { Number of spindle } \\
\text { stromal cells, /HPF }\end{array}$ & Regenerated epithelium & Subepithelial fibrous tissue & Muscularis propria \\
\hline \multicolumn{7}{|l|}{ TA group } \\
\hline 1 & 2 & 1 & 189 & 85 & 185 & 2672 \\
\hline 2 & 1 & 1 & 126 & 162 & 15 & 2817 \\
\hline 3 & 3 & 1 & 270 & 224 & 1151 & 1689 \\
\hline 4 & 2 & 3 & 369 & 209 & 634 & 5005 \\
\hline 5 & 2 & 1 & 252 & 132 & 47 & 3986 \\
\hline 6 & 2 & 2 & 147 & 231 & 239 & 2847 \\
\hline 7 & 2 & 1 & 363 & 67 & 81 & 1582 \\
\hline Median (range) & - & - & $252(126-369)$ & $162(66-231)$ & $185(15-1151)$ & $2817(1582-5005)$ \\
\hline \multicolumn{7}{|l|}{ Non-TA group } \\
\hline 1 & 2 & 1 & 177 & 81 & 860 & 1937 \\
\hline 2 & 2 & 3 & 462 & 410 & 871 & 1959 \\
\hline 3 & 2 & 1 & 144 & 144 & 886 & 2764 \\
\hline 4 & 1 & 1 & 63 & 112 & 779 & 1325 \\
\hline 5 & 1 & 1 & 102 & 114 & 810 & 1487 \\
\hline 6 & 3 & 1 & 339 & 261 & 753 & 2701 \\
\hline 7 & 2 & 2 & 243 & 163 & 887 & 1902 \\
\hline Median (range) & - & - & $177(63-462)$ & $144(82-410)$ & 860 (753-887) & $1937(1325-2764)$ \\
\hline$P$ value & 1 & 1 & 0.443 & 0.898 & 0.030 & 0.125 \\
\hline
\end{tabular}

Italic values indicate significance of $p$ value $(P<0.05)$

${ }^{a}$ Density of collagen fiber: 1 , Sparse proliferation of collagen fibers with edema; 2, Dense proliferation of collagen fiber with mild edema; 3 , Dense proliferation of collagen fiber without edema

${ }^{b}$ Density of smooth muscle cells: 1 , None or small amount of smooth muscle fibers mixed with collagen fibers; 2 , Moderate mixture of smooth muscle fibers but less than collagen fibers; 3 , Almost the same amount of smooth muscle fibers as collagen fibers

No. case number, $H P F$ high power field, $T A$ triamcinolone acetonide

healing process of ESD ulcer, the proliferation of myofibroblasts, which are dedifferentiated from myocytes of the muscularis propria and arranged in a parallel fashion in the submucosal layer, may play an important role in esophageal stricture formation. They concluded that TA injection may modify these processes to prevent esophageal strictures.

In our study, characteristic findings such as fibrosis, thickness, and inflammatory cell infiltration in the MP were not obtained, but the thinning of the SF was observed in the TA injection group. These characteristic changes that occurred in the subepithelial layer may be consistent with those observed in the aforementioned basic studies using animal models. These two animal studies [32, 33] suggested that the fibrosis in the submucosal layer of the ESD ulcer scar is related to stricture formation.

The muscularis propria of the pig esophagus is a striated muscle, which may not necessarily be similar to the smooth muscle of the human esophagus, but it may be suggestive of its role in esophageal stricture formation.
Further, studies have been conducted in the human gastrointestinal tract for histopathological examination of stenosis formation in Crohn's disease [34]. Suekane et al. reported that accumulation of fibrous tissue was observed in the submucosal layer in all intestinal stenotic lesions in Crohn's disease. They concluded that the proliferation and migration of moderately differentiated intestinal smooth muscle cells from the muscular layers are the underlying pathological mechanisms in stricture formation.

Assuming that the above mechanism is instrumental in the human esophagus, esophageal stenosis may be associated with an increase in myofibroblast dedifferentiation from the muscular layer and proliferation of fibrous tissue in the scar area. It is highly probable that local steroid injection is involved in the suppression of these processes, which may be attributed for the thinning of The SF.

In Nonaka et al.'s study, the longest period from ESD to esophagectomy was 8 weeks. In contrast, in our study, the interval between ESD and surgery was $103.9 \pm 35.0$ days, which was longer than the observation period of Nonaka 
et al.'s report. Therefore, the number of immature myofibroblasts might have decreased or disappeared when observing surgical specimens under a microscope. MT staining showed that there was no difference in the number of spindle stromal cells, and myofibroblasts between the two groups. Additionally, owing to the long duration between ESD and esophagectomy, it was difficult to observe the state of myofibroblasts.

Since desmin-positive cells are mixed in the fibrotic tissue of the scar, myofibroblast migration and proliferation of fibrous tissue due to dedifferentiation from the MP might have occurred in the human esophagus. Although this may be consistent with the findings of Nonaka et al. [33] and Suekane et al. [34], further investigation is needed to obtain conclusive results.

This study has some limitations. First, this is a retrospective study conducted in a single institute using past pathological specimens. However, in studies using human specimens, ESD cannot be performed on the premise of additional esophagectomy; thus, examining histological changes over time prospectively appears impossible. Second, the number of cases examined was small because cases of esophagectomy performed as an additional treatment after ESD are few. In our institution, only 4.4\% (13 of 297 cases) of the patients underwent additional esophagectomy after ESD during the current observation period. This is a small-scale retrospective study comparing seven patients in the TA group with six in the Non-TA group, and this is preliminary study. Third, the site where TA was actually injected could not be identified histologically despite careful microscopic observations by a skilled pathologist. Roques et al. [35] reported that the injected TA remains locally active for 3-4 weeks. In the present study, the interval from TA injection immediately after ESD to additional surgery was much longer (103.9 \pm 35.0 days) than 3-4 weeks, so it was difficult to detect TA injection site histologically.

In conclusion, our study shows a characteristic histopathological finding of thinning of The SF in cases with local TA injection after esophageal ESD. To the best of our knowledge, this is the first histological study using human esophagectomy specimens about TA injection after esophageal ESD. Further investigation such as immunohistological study may help elucidate the basic mechanism on how steroid prevents esophageal stricture after ESD.

Supplementary Information The online version contains supplementary material available at https://doi.org/10.1007/s10388-021-00818-0.

Acknowledgements We are grateful to Masako Akiyama for her precise advice for statistical analyses.

\section{Compliance with ethical standards}

Ethical Statement This study was a single-center, retrospective study wherein only the medical records and resected specimens that had already been obtained from the patients were used; therefore, we did not have written consent from the participants. However, all the patients were informed in writing at the time of treatment that the specimens obtained from the treatment may be used in future clinical research, and consent was signed by all patients. A notice was posted on the website of the Department of Gastrointestinal Surgery, which provided an opportunity to the patients to give informed consent or refuse from research participation. This study was approved by the Tokyo Medical and Dental University School of Medicine Ethics Review Board (approval number: M2018-191), and all the procedures were performed in accordance with the principles of Declaration of Helsinki, 1964 and its later amendments.

Conflict of interest Yudai Kawamura, Kenro Kawada, Takashi Ito, Katsumasa Saito, Naoto Fujiwara, Takuya Okada, Akihiro Hoshino, Yutaka Tokairin, Yasuaki Nakajima, Tatsuyuki Kawano, Masanori Tokunaga, and Yusuke Kinugasa have no conflicts of interest to declare.

Open Access This article is licensed under a Creative Commons Attribution 4.0 International License, which permits use, sharing, adaptation, distribution and reproduction in any medium or format, as long as you give appropriate credit to the original author(s) and the source, provide a link to the Creative Commons licence, and indicate if changes were made. The images or other third party material in this article are included in the article's Creative Commons licence, unless indicated otherwise in a credit line to the material. If material is not included in the article's Creative Commons licence and your intended use is not permitted by statutory regulation or exceeds the permitted use, you will need to obtain permission directly from the copyright holder. To view a copy of this licence, visit http://creativecommons.org/licenses/by/4.0/.

\section{References}

1. Freddie B, Ferlay J, Jemal A, et al. Global cancer statistics 2018: GLOBOCAN estimates of incidence and mortality worldwide for 36 cancers in 185 countries. CA Cancer J Clin. 2018;68:94-424.

2. Oyama T, Tomori A, Miyata Y, et al. Endoscopic submucosal dissection of early esophageal cancer. Clin Gastroenterol Hepatol. 2005;3:S67-70.

3. Shah PM, Gerdes H. Endoscopic options for early stage esophageal cancer. J Gastrointest Oncol. 2015;6:20-30.

4. Plum PS, Holscher AH, Pacheco Godoy K, et al. Prognosis of patients with superficial T1 esophageal cancer who underwent endoscopic resection before esophagectomy-a propensity scorematched comparison. Surg Endosc. 2018;32:3972-80.

5. Tsujii Y, Nishida T, Nishiyama O, et al. Clinical outcomes of endoscopic submucosal dissection for superficial esophageal neoplasms: a multicenter retrospective cohort study. Endoscopy. 2015;47:775-83.

6. Kato H, Tachimori Y, Itabashi M, et al. Superficial esophageal carcinoma: surgical treatment and the results. Cancer. 1990;66:2319-23.

7. Kato H, Tachimori Y, Ochiai A, et al. Cervical, mediastinal, and abdominal lymph node dissection (three-field dissection) for superficial carcinoma of the thoracic esophagus. Cancer. 1993;72:2879-82. 
8. Ono S, Fujishiro M, Omata M, et al. Predictors of postoperative stricture after esophageal endoscopic submucosal dissection for superficial squamous cell neoplasms. Endoscopy. 2009;41:661-5.

9. Katada C, Muto M, Manabe T, et al. Esophageal stenosis after endoscopic mucosal resection of superficial esophageal lesions. Gastrointest Endosc. 2003;57:165-9.

10. Kochhar R, Makharia GK. Usefulness of intralesional triamcinolone in treatment of benign esophageal strictures. Gastrointest Endosc. 2002;56:829-83.

11. Hashimoto S, Kobayashi M, Aoyagi Y, et al. The efficacy of endoscopic triamcinolone injection for the prevention of esophageal stricture after endoscopic submucosal dissection. Gastrointest Endosc. 2011;74:1389-93.

12. Hanaoka N, Ishihara $\mathrm{R}$, Takeuchi $\mathrm{Y}$, et al. Intralesional steroid injection to prevent stricture after endoscopic submucosal dissection for esophageal cancer: a controlled prospective study. Endoscopy. 2012;44:1007-11.

13. Rajan E, Gostout C, Pasricha P, et al. Widespread endoscopic mucosal resection of the esophagus with strategies for stricture prevention: a preclinical study. Endoscopy. 2005;37:1111-5.

14. Yamashita T, Uedo N, Ishii H, et al. Delayed perforation after intralesional triamcinolone injection for esophageal stricture following endoscopic submucosal dissection. Endoscopy. 2013;45:E92.

15. Matsuda Y, Kataoka N, Makimoto S, et al. Delayed esophageal perforation occurring with endoscopic submucosal dissection: A report of two cases. World J Gastrointest Surgery. 2015;7:123-7.

16. Kanda Y. Investigation of the freely available easy-to-use software 'EZR' for medical statistics. Bone Marrow Transplant. 2013;48:452-8.

17. Oliveira JF, Moura EG, Bernardo WM, et al. Prevention of esophageal stricture after endoscopic submucosal dissection: a systematic review and meta-analysis. Surg Endosc. 2016;30:2779-91.

18. Ezoe Y, Muto M, Horimatsu T, et al. Efficacy of preventive endoscopic balloon dilation for esophageal stricture after endoscopic resection. J Clin Gastroenterol. 2011;45:222-7.

19. Shi P, Ding X. Progress on the prevention of esophageal stricture after endoscopic submucosal dissection. Gastroent Res Pract. 2018;2018:1696849.

20. Sakaguchi Y, Fujishiro M, Koike K, et al. Polyglycolic acid sheets with fibrin glue can prevent esophageal stricture after endoscopic submucosal dissection. Endoscopy. 2015;47:336-40.

21. Li L, Linghu E, Chai N, et al. Clinical experience of using a novel self-help inflatable balloon to prevent esophageal stricture after circumferential endoscopic submucosal dissection. Dig Endosc. 2019;31:453-9.

22. Yu M, Tan Y, Liu D. Strategies to prevent stricture after esophageal endoscopic submucosal dissection. Ann Transl Med. 2019;7:271.

23. Yamaguchi N, Isomoto H, Nakayama T, et al. Usefulness of oral prednisolone in the treatment of esophageal stricture after endoscopic submucosal dissection for superficial esophageal squamous cell carcinoma. Gastrointest Endosc. 2011;73:1115-21.

24. Shibagaki K, Ishimura N, Kinoshita Y, et al. Esophageal triamcinolone acetonide-filling method: a novel procedure to prevent stenosis after extensive esophageal endoscopic submucosal dissection (with videos). Gastrointest Endosc. 2018;87:380-9.

25. Nagami Y, Shiba M, Arakawa T, et al. Locoregional steroid injection prevents stricture formation after endoscopic submucosal dissection for esophageal cancer: a propensity score matching analysis. Surg Endosc. 2016;30:1441-9.

26. Okamoto K, Matsui S, Kudo M, et al. Clinical analysis of esophageal stricture in patients treated with intralesional triamcinolone injection after endoscopic submucosal dissection for superficial esophageal cancer. Oncology. 2017;93(Suppl. 1):9-14.

27. Hanaoka N, Ishihara R, Ishii H, et al. Refractory strictures despite steroid injection after esophageal endoscopic resection. Endosc Int Open. 2016;4:E354-9.

28. Griffith BH. The treatment of keloids with triamcinolone acetonide. J Plast Reconstr Surg. 1966;38:202-8.

29. Wong T-S, Li JZ-H, Chen S, Chan JY-W, Gao W. The efficacy of triamcinolone acetonide in keloid treatment: a systematic review and meta-analysis. Front Med (Lausanne). 2016;3:71.

30. Cruz NI, Korchin L. Inhibition of human keloid fibroblast growth by isotretinoin and triamcinolone acetonide in vitro. Ann Plast Surg. 1994;33:401-5.

31. Boyadjiev C, Popchristova E, Mazgalova J. Histomorphologic changes in keloids treated with Kenacort. J Trauma. 1995;38:299-302.

32. Honda M, Nakamura T, Hashimoto Y, et al. Process of healing of mucosal defects in the esophagus after endoscopic mucosal resection: histological evaluation in a dog model. Endoscopy. 2010;42:1092-5.

33. Nonaka K, Miyazawa M, Kita H, et al. Different healing process of esophageal large mucosal defects by endoscopic mucosal dissection between with and without steroid injection in an animal model. BMC Gastroenterol. 2013;13:72.

34. Suekane T, Ikura Y, Watanabe K, et al. Phenotypic change and accumulation of smooth muscle cells in strictures in Crohn's disease: relevance to local angiotensin II system. J Gastroenterol. 2010;45:821-30.

35. Roques $\mathrm{C}$, Teot $\mathrm{L}$. The use of corticosteroids to treat keloids: a review. Int J Low Extrem Wounds. 2008;7:137-45.

Publisher's Note Springer Nature remains neutral with regard to jurisdictional claims in published maps and institutional affiliations. 